\title{
Der Übergang auf weiterführende Schulen aus rechtlicher und aus pädagogischer Sicht
}

\section{Zusammenfassung}

Kaum ein Bereich schulischer Entscheidungen war und ist rechtlich so umstritten und pädagogisch so anspruchsvoll wie die Übergangsmodalitäten nach dem Ende der Grundschulzeit auf eine weiterführende Schule. Mit diesem Beitrag werden zunächst die juristischen Dimensionen des Verfahrens in ihrer historischen Genese mit Fokus auf das Bundesland Hessen beschrieben und rechtlich eingeordnet. Anschließend werden jenseits der bloßen Übergangsempfehlung die Phasen eines gelingenden Übergangs als pädagogische Herausforderung mit Blick auf die Frage skizziert, wie dieser gelingen kann. Es zeigt sich, dass sich die Frage nach Chancengleichheit im Kontext von Übergangsregelungen nicht auf die rechtlichen Vorgaben reduzieren lässt. Vielmehr realisieren sich Übertrittsentscheidungen erst mit dem faktischen Übertritt, der pädagogisch zu begleiten ist, damit er erfolgreich bewältigt werden kann.

Schlüsselwörter: Übergänge, Schulrecht, Hessen

\section{The Transition to Secondary Schools from a Legal and a Pedagogical Point of View}

\begin{abstract}
Hardly any area of school decisions was and is legally so controversial and pedagogically so demanding as the transition modalities from primary to secondary school. This article first describes the legal dimensions of the proceedings in their historical genesis with a focus on the federal state of Hesse. Then, beyond the mere transition recommendation, the phases of a successful transition are outlined as an educational challenge. It turns out that the question of equal opportunities in the context of transitional regulations cannot be reduced to legal requirements. Rather, transfer decisions are only realized within the actual transfer, which must be accompanied pedagogically so that it can be successfully managed.
\end{abstract}

Keywords: transitions, educational law, Hesse 
Der Übergang auf weiterführende Schulen aus rechtlicher und aus pädagogischer Sicht |

\section{Die juristischen Dimensionen des Übergangsverfahrens}

\subsection{Entwicklungen der Regelungen im Bundesland Hessen}

Um die heutige Rechtslage einordnen zu können, ist ein kurzer Rückblick auf frühere rechtliche Regelungen hilfreich, da dieser zeigt, welche Modalitäten es gab und wie es zur heutigen Regelungspraxis gekommen ist.

In der Zeit von 1949 bis 1955 war das Übertrittsverfahren in Hessen lediglich durch verwaltungsinterne Regeln festgelegt, nach denen die abgebende (Volks-)Schule aufgrund der Ergebnisse eines landeseinheitlichen Tests in der Klasse 4 entschied, ob ein Schüler bzw. eine Schülerin an einem Ausleseverfahren zum Besuch einer weiterführenden Schule (Gymnasium, Mittelschule) teilnehmen durfte. Dieser Test wurde im Laufe der Jahre durch die Noten des Zwischenzeugnisses der Klasse 4 und die Beurteilung der Klassenlehrkraft ersetzt. ${ }^{1}$

In dem Ausleseverfahren musste mindestens ein Notendurchschnitt von 2,5 erreicht werden, um in die gewünschte weiterführende Schule aufgenommen werden zu können. Das erste Jahr an der weiterführenden Schule war zudem als Probejahr ausgestaltet, sodass Schülerinnen und Schüler bei Unterschreiten der geforderten schulischen Leistung an die Volksschule zurücküberwiesen werden konnten (vgl. hierzu Hess-Köller Anm. 1 zu $\$ 5$ a HSchVwG).

Mit Verwaltungsvorschrift vom 19.12.1960 (ABl., 1961, S. 8 ff.) wurden die Bestimmungen zum Übergang innerhalb der allgemeinbildenden Schulen in Hessen erstmals rechtssatzmäßig geregelt. Diese Bestimmungen, die mit Erlass vom 31.05.1972 (ABl., S. 599) erneut in Kraft gesetzt worden $\operatorname{sind}^{2}$, hatten vorgesehen, dass die Eltern nach einer regelhaft vorangegangenen Beratung mit der Klassenlehrkraft über die Schulleitung der Volksschule bei der Schulleitung der gewünschten weiterführenden Schule die Aufnahme für ihr Kind beantragen konnten. Seitens der Volksschule wurden diesem Antrag die letzten beiden Zeugnisse, der Schüler- bzw. Schülerinnenbogen sowie ein Gutachten der Klassenlehrkraft oder der Klassenkonferenz zur Bildungsfähigkeit und dem Arbeitswillen des Schülers bzw. der Schülerin beigefügt, in dem auch zur Eignung für die gewünschte weiterführende Schule Stellung genommen wurde. Für den Fall der Übereinstimmung von Elternwunsch und schulischer Einschätzung war die Aufnahme an der gewünschten Schule vorgesehen. Im Divergenzfall musste sich der Schüler bzw. die Schülerin, sofern die Eltern ihren Antrag aufrechterhielten, einem mehrtägigen Ausleseverfahren unterziehen, das aus einem sechstägigen Probeunterricht in den Fächern Deutsch, Rechnen (sic) und Heimat-

1 Vgl. Hess-Köller Anm. 1 zu $₫ 5$ a HSchVwG.

2 Verwaltungsvorschriften treten in Hessen nach 10 Jahren automatisch außer Kraft und sind dann erneut zu erlassen. 
kunde bestand und in dessen Verlauf eine Nacherzählung, eine Nachschrift und zwei Rechenarbeiten anzufertigen waren.

Mit der auf $\$ 58$ Abs. 2 Nr. 2 HSchVwG fußenden Verordnung über die Übergänge innerhalb der allgemeinbildenden Schulen vom 10.03.1983 (ABl., S. 293) wurde nach Einzug des Gesetzesvorbehalts in das Schulrecht die bisherige Regelung in einer rechtsstaatlichen Grundsätzen entsprechenden Weise mit geringen inhaltlichen Veränderungen fortgeführt. Nunmehr war vorgesehen, dass die Eltern ihr Kind über die Grundschulleitung bei der Leitung der gewünschten weiterführenden Schule (d.h. Realschule, Gymnasium oder vergleichbarer Schulzweig einer schulformbezogenen Gesamtschule) anmeldeten. Sofern die Klassenkonferenz der Grundschule ein Kind nur als bedingt geeignet für die gewünschte Schulform beurteilt hatte, war nach $\$ 2$ Abs. 2 Satz 3 und $\$ 3$ Abs. 4 der Übergangsverordnung ein Gutachten zur Bildungsfähigkeit, zu den Leistungen und zum Arbeitswillen des Kindes anzufertigen. In diesen Fällen war nach $\$ 4$ Abs. 1 ÜbergangsVO ein fünftägiger Probeunterricht mit mindestens drei Stunden pro Tag vorgesehen, in dessen Verlauf das Kind drei schriftliche Arbeiten anzufertigen hatte (davon je eine in den Fächern Deutsch und Mathematik).

Mit der Verordnung vom 05.02.1988 (ABl., S. 39) wurde die bisherigen Rechtslage dahingehend verändert, dass nunmehr zunächst die abgebende Schule in einem Eignungsgutachten feststellen sollte, für welchen weiterführenden Bildungsgang das Kind geeignet erscheint, ehe die Erziehungsberechtigten ihre Entscheidung treffen. Bei Abweichungen zwischen Eignungsgutachten und elterlicher Wahlentscheidung sollte das Ergebnis eines Probeunterrichts über die weitere Schullaufbahn entscheiden.

Diese Verordnung ist vom Hessischen Verwaltungsgerichtshof (HVGH) mit einem in einem Normenkontrollverfahren nach $\$ 47$ VwGO ergangenen Beschluss vom

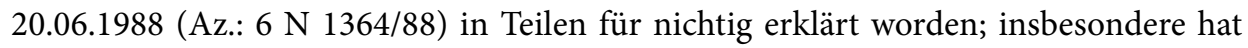
es der HVGH für unverhältnismäßig angesehen, dass in einer so wichtigen Frage wie der Entscheidung über die weitere Schullaufbahn eines Kindes der Staat noch vor den Eltern das „erste Wort“ haben sollte. Darüber hinaus hat der HVGH die Regelung auch wegen ihres Eingriffscharakters mit der Lehre vom Gesetzesvorbehalt für unvereinbar angesehen, da sie auf keiner gesetzlichen Grundlage beruhe.

Der hessische Gesetzgeber hat unter Beachtung dieser Rechtsprechung des HVGH durch die Einfügung des $\$$ a HSchVwG vom 17.03.1989 (GVBl. I, S. 101) und der darauf fußenden Verordnung über die Übergänge innerhalb der allgemeinbildenden Schulen bis zur Jahrgangsstufe 10 vom 05.05.1989 (ABl., S. 354) sowohl die notwendige rechtssatzmäßige Regelung erlassen als auch die vom HVGH geforderte Reihenfolge der Entscheidungen von Eltern und Schule bezüglich der Schullaufbahnentscheidung getroffen. 
Der Übergang auf weiterführende Schulen aus rechtlicher und aus pädagogischer Sicht |

Mit dem Gesetz zur „Einführung der freien Wahl der Bildungswege und zur vorläufigen Regelung der Übergänge nach der Grundschule und der Förderstufe“ vom 13.06.1991 (GVBl. I, S. 181) wurde das Primat der elterlichen Entscheidung wiederhergestellt. Die nunmehr geltende Regelung des $₫ 5$ a Abs. 2 HSchVwG gestand den Eltern die Wahl der weiterführenden Schulart zu; sofern die Klassenkonferenz diese Wahlentscheidung befürwortete, war das Kind an dieser aufzunehmen. Im Divergenzfall musste die Klassenkonferenz ihren Beschluss schriftlich begründen. Sofern die Eltern trotzdem an ihrer Wahlentscheidung festhielten, war die aufnehmende Schule - zumal es in Hessen nie eine Versetzung auf Probe gab - hieran gebunden.

In entsprechender Weise ist diese Regelung in $\$ 77$ des am 17.06.1992 verabschiedeten und zum 01.08.1993 in Kraft getretenen HSchG (GVBl. I, S. 233 ff.) aufgenommen worden.

Mit der Schulgesetznovelle des Jahres 1999 (vom 30.06.1999, GVBl. I, S. 354) wurde das Verfahren dahingehend geändert, dass die Grundschule zunächst die Erziehungsberechtigten nach ihren Vorstellungen fragen musste und danach eine eigene Einschätzung in Gestalt einer Bildungsempfehlung abgab.

Sofern Elternwunsch und schulische Einschätzung übereinstimmten, konnte das Kind seinen schulischen Werdegang ohne Einschränkungen entsprechend fortsetzen. Für den Fall, dass Elternwunsch und Einschätzung der Grundschule nicht übereinstimmten, war die Grundschule verpflichtet, den Eltern eine weitere Beratung anzubieten, zu deren Entgegennahme oder Beachtung die Erziehungsberechtigten jedoch nicht verpflichtet waren. Vielmehr stand es den Erziehungsberechtigten frei, nunmehr in eigener Zuständigkeit über den weiteren Bildungsgang ihres Kindes zu entscheiden. Die Aufnahme an einer Schule des von den Erziehungsberechtigten gewünschten Bildungsgangs - nicht allerdings an einer konkreten Schule dieses Bildungsgangs - erfolgte dann unter dem Vorbehalt, dass die Schule bis zum Ende des 5. Schuljahres berechtigt war, bei Nichterfüllung der Leistungsvoraussetzungen eine Querversetzung des Kindes in einen weniger anspruchsvollen Bildungsgang gemäß $₫ 75$ Abs. 3 HSchG zu beschließen. Mit der Versetzung in die Jahrgangsstufe 6 des gewünschten Bildungsgangs erfolgte die vorerst endgültige Zuordnung zu dem gewählten Bildungsgang.

Diese Form des Übergangs in die Sekundarstufe I ist mit der Novellierung des Schulgesetzes vom 14.07.2011 (GVBl. I, S. 265) wieder aufgegeben worden. Seitdem gilt in Hessen wieder das Letztentscheidungsrecht der Erziehungsberechtigten, denen seitens der Grundschule lediglich eine rechtlich unverbindliche Einschätzung zum weiteren schulischen Werdegang des Kindes zur Verfügung gestellt wird. 
Vergleichbare Übergangsregelungen ohne Letztentscheidungsrecht des Staates haben sich in der Folgezeit in den meisten Bundesländern - mit Ausnahme der Freistaaten Bayern und Sachsen - durchgesetzt (vgl. Art. 44 BayEUG; $\$ 34$ SächsSchG).

\subsection{Rechtliche Einordnung}

Bei der rechtlichen Einordnung der Modalitäten des Übergangs auf weiterführende Schulen ist von der verfassungsrechtlich gesicherten Grundposition auszugehen, dass die Entscheidung über den nach der Grundschule zu besuchenden Bildungsgang sowohl zentraler Bestandteil des in Art. 6 Abs. 2 GG geschützten Erziehungsrechts der Eltern ist, als auch zum selbstverständlichen Inhalt der dem Staat nach Art. 7 Abs. 1 GG obliegenden Organisations- und Aufsichtsbefugnis gehört.

Der praktische Ausgleich dieser beiden grundgesetzlich einander gleichberechtigt gegenüberstehenden Positionen hat nach der Rechtsprechung des BVerfG (vgl. grundlegend BVerfG vom 06.12.1972, AS 34, $165 \mathrm{ff}$.) nach dem Grundsatz der praktischen Konkordan $\mathrm{z}^{3} \mathrm{zu}$ erfolgen. Insoweit ist bis heute unumstritten, dass der Staat berechtigt ist, den Übergang nach Klasse 4 bzw. 6 in eine weiterführende Schule letztverbindlich selbst $\mathrm{zu}$ regeln. Hierfür ist lediglich erforderlich, dass eine gesetzliche Grundlage vorhanden ist, in der die grundlegenden Voraussetzungen für den Übergang geregelt sind und des Weiteren verfahrensrechtlich sichergestellt ist, dass die Erziehungsberechtigten als erste ihre Vorstellungen über den weiteren Bildungsgang ihres Kindes äußern können. Der Staat ist in diesem Verfahren erst in zweiter Linie berechtigt zu entscheiden, ob er die Wahlentscheidung der Erziehungsberechtigten teilt oder nicht. Das in den meisten Bundesländern zwischenzeitlich vorgesehene Letztentscheidungsrecht der Erziehungsberechtigten ist nach dieser verfassungsrechtlichen Ausgangslage zwar nicht geboten, aber jedenfalls zulässig und erscheint als pragmatische Lösung. Möglicherweise auftretende Dissensfälle, die sich im weiteren schulischen Werdegang als offensichtliche Fehlentscheidung herausstellen, können entweder durch gezieltere Beratungen gelöst oder als Einzelfälle vom System aufgefangen und ausgehalten werden.

Im Sinne einer Liberalisierung der Übergangsmodalitäten könnte daher bei Vorhandensein eines funktionierenden Beratungssystems der Grundschule auf weitergehende Instrumente staatlicher Lenkungsentscheidungen grundsätzlich verzichtet werden.

3 Praktische Konkordanz bezeichnet die notwendige Abwägung zweier miteinander kollidierender grundrechtlicher Güter (vgl. schon BVerfGE 41, 29 (103)). 
Der Übergang auf weiterführende Schulen aus rechtlicher und aus pädagogischer Sicht |

\section{2 Übergangsprozesse von der Grundschule in die weiterführende Schule als pädagogische Aufgabe - ein Kommentar ${ }^{4}$}

Der Übergang von der Grundschule in weiterführende Schulen lässt sich nicht auf bloße, formale Übergangsempfehlungen reduzieren. Im Sinne der Liberalisierung der Übergangsmodalitäten ist eine stärkere pädagogische ,Einhegung des Übergangsprozesses unerlässlich. An die Stelle der rechtlichen Steuerung tritt die pädagogische Beratung und Begleitung der Schülerinnen und Schüler. Vor diesem Hintergrund handelt es sich beim Übergang um eine Phase, die mindestens ab der 5., idealerweise schon ab dem 2. Schulhalbjahr der 4 . Klasse beginnt und pädagogisch begleitet wird. Diese Phase stellt für viele Schülerinnen und Schüler eine besondere Herausforderung dar. Neue Klassenkameradinnen und Klassenkameraden, ein neuer Schulweg, ein größeres und unter Umständen unübersichtliches Gebäude bzw. Schulgelände, eine größere Anzahl von Lehrkräften, die in der Klasse unterrichten u.a.m. können sich als große Hürden zu Beginn des ersten Schuljahres in der Sekundarstufe erweisen.

Im Regelfall haben Sekundarstufenschulen im Grundschulbereich verschiedene Zulieferschulen aus Stadtteilen bzw. benachbarten Gemeinden. Daher ist es notwendig, sich gemeinsam dieser für die Schülerinnen und Schüler so wichtigen Phase anzunehmen und bereits das zweite Halbjahr des 4. Schuljahres in die Planungen einzubeziehen. Nur so kann es gelingen, den Übergang zwischen den betroffenen Grundschulen und den Sekundarstufenschulen gut vorzubereiten und in der Stufe 5/6 angemessen $\mathrm{zu}$ begleiten.

Eine in diesem Sinne geeignete Konzeption könnte sich in drei Phasen gliedern:

Phase 1: Zeit der Entscheidungsfindung zum Übergang (etwa bis zu den Osterferien im 4. Schuljahr)

Diese Phase betrifft die Entscheidungsfindung der Eltern für den zukünftigen Werdegang ihrer Kinder. Hier finden Informationsveranstaltungen für die und Kontakte mit den Eltern, aber auch das Sammeln erster Erfahrungen durch die Schülerinnen und Schüler statt. Hierfür eignen sich unter anderem:

- Tage der offenen Tür,

- Mitmachunterricht und

- Beratungsgespräche.

4 Die nachfolgenden Ausführungen stützen sich auf die Erfahrungen und Schulleitungspraxis des Verfassers, Heinz Kipp. 
Phase 2: Zeit nach der Entscheidung und vor dem eigentlichen Übergang (etwa Ostern bis zu den Sommerferien)

Für die Sekundarstufenschule könnten folgende Aktivitäten im Rahmen eines Kennenlerntages angeboten werden:

- Teilnahme am Regelunterricht;

- Teilnahme an besonderen Unterrichtsveranstaltungen, z. B. sportliche Aktivitäten, Unterricht mit digitalen Medien, Dichterlesungen, Darstellendes Spiel etc.;

- Kennenlernen der zukünftigen Klassen- bzw. Fachlehrkräfte;

- Gestaltung eines gemeinsamen Wandertags, eines Klassenfests o. ä.;

- Durchführung eines Kennenlerntages zwischen den Lehrkräften der weiterführenden Schule und Grundschullehrkräften des vierten Schuljahres.

Um den Übergang zu erleichtern und ihn fließender zu gestalten, sollte die neue Schule ferner in Kontakt zu den Grundschulen ihres Einzugsgebietes stehen. So können beispielsweise im 1. Schulhalbjahr Treffen mit den Lehrkräften der abgebenden Grundschulen und den Lehrkräften der Sekundarstufenschulen stattfinden. Auf diesen Zusammenkünften tauschen sich die Lehrkräfte zum einen über einzelne Schülerinnen und Schüler aus. Die Kolleginnen und Kollegen der Grundschule erfahren bei dieser Gelegenheit, wie sich ihre ehemaligen Schülerinnen und Schüler entwickeln. Die Lehrkräfte der weiterführenden Schule erhalten wichtige Informationen über ihre neuen Fünftklässlerinnen und Fünftklässler. Zum anderen erfolgt ein fachlicher Austausch. Hier kann beispielsweise über die Gestaltung und Bewertung von Klassenarbeiten gesprochen oder das Anspruchsniveau von Schulbüchern verglichen werden. Um den Austausch effektiver zu gestalten, sollten sich die jeweiligen Fachsprecherinnen und Fachsprecher der einzelnen Schulen zu einem gesonderten Termin zusammenfinden und die dort getroffenen Absprachen an ihre Kolleginnen und Kollegen weitergeben.

Phase 3: Zeit nach dem Übergang (nach den Sommerferien bzw. nach der Einschulung)

Für die Schülerinnen und Schüler der 5. Klassen beginnt das Schuljahr in der Regel mit einer sogenannten ,Einführungsphase` oder auch ,Orientierungsphase', die sich über das erste Schulhalbjahr erstrecken kann. Ziel ist es, den Schülerinnen und Schülern die Integration in die weiterführende Schule und vor allem in die jeweils neue Klasse zu erleichtern. Auch sollen dabei unterschiedliche Leistungsstände kompensiert und Grundlagen für eine erfolgreiche Arbeit an der Schule geschaffen werden. In den ersten Tagen und Wochen des Schuljahres werden die Schülerinnen und Schüler mit der Organisation des Alltagsbetriebs an der besuchten Schule behutsam vertraut gemacht. Sie erfahren, wie der Unterrichtsablauf gestaltet ist und an wen sie sich bei Fragen wenden können. 
Der Übergang auf weiterführende Schulen aus rechtlicher und aus pädagogischer Sicht |

Eine wichtige Funktion der Einführungsphase bzw. Orientierungsphase ist die Förderung des sozialen Lernens sowie der Gruppenbildungsprozesse in den Klassen. So können besonders zu Beginn Kennenlernspiele, gemeinschaftliche Lösungen von Aufgaben und kleine gemeinsame Unternehmungen das Einleben in das neue Umfeld fördern. Über die Gestaltung eines positiven Klassenklimas hinaus sollte eine allmähliche Identifikation mit der gesamten Schule angestrebt werden.

Zusätzlich kann der Übergang von der Grundschule in die weiterführende Schule erleichtert werden, indem

- eine tragfähige Klassengemeinschaft entsteht bzw. wächst;

- Schülerinnen und Schüler zu selbstständigem Arbeiten angeleitet werden;

- Teamarbeit gefördert wird;

- Schülerinnen und Schüler in all ihren Bedürfnissen ernst genommen werden;

- soziales Lernen unterstützt und gefördert wird.

Weitere Möglichkeiten bieten zum Beispiel auch die Kontingentstundentafeln, die für jede Schulart festlegen, wie viele Jahreswochenstunden insgesamt in den Schuljahren bis zum Abschluss des Bildungsgangs zu erteilen sind. Wie diese Jahreswochenstunden jedoch auf die einzelnen Klassenstufen verteilt werden, entscheiden die einzelnen Schulen. Sie erhalten damit pädagogischen Freiraum und können die Verteilung der Stunden nutzen, um Schwerpunkte zu setzen und die Schulkonzepte zu gestalten. Aber auch die stärkere Einbindung der Planungen in an der Schule bereits vorhandene Konzepte kann vielfältige Möglichkeiten eröffnen.

Zusammenfassend kann festgestellt werden, dass es für die Planung des Übergangs von Schülerinnen und Schülern von der Grundschule in die weiterführende Schule von großer Bedeutung ist, dass bei Grundschule und weiterführender Schule gleichermaßen das Bewusstsein vorhanden ist, dass isolierte Vorhaben, die nicht auf eine Verzahnung abzielen, keine große Wirkung entfalten werden. Damit Kinder den Übergang nicht als tiefen Einschnitt in ihrer persönlichen Schullaufbahn erfahren, ist somit ein gemeinsames und abgestimmtes Vorgehen von abgebender und aufnehmender Schule erforderlich. Rückbezogen auf die Frage nach Chancengleichheit zeigt sich, dass die Frage nach der Herstellung materieller Chancengleichheit zwar von den Modalitäten der Übergangsempfehlung bedingt wird, sich jedoch nicht auf diese reduzieren lässt. Realisiert wird sie in der konkreten Ausgestaltung und pädagogischen Begleitung des Übertritts.

Hinweis der Redaktion: Als Hilfestellung sind die wesentlichen rechtlichen Regelungen, auf die in den Beiträgen des Heftes Bezug genommen wird, in einem das Heft begleitenden Online-Anhang abgedruckt. Er ist unter folgendem Link abrufbar: https://www.waxmann. com/artikelART104608 
| Wolfgang Bott \& Heinz Kipp

Wolfgang Bott, Dr., geb. 1949, Ministerialrat i. R., Geschäftsführer von HLP-Bildungsmanagement, Frankfurt am Main

E-Mail: info@drwolfgangbott.de

Korrespondenzadresse: Im Klaf 21, 65207 Wiesbaden

Heinz Kipp, geb. 1949, Leitender Regierungsdirektor a.D., Geschäftsführer HLP BildungsManagement, Frankfurt am Main

E-Mail: heinz.kipp@hlp-bildung.de

Korrespondenzadresse: Narzissenstraße 9, 61169 Friedberg 